\title{
PATH PLANNING AND POSITIONING TECHNOLOGY OF INTELLIGENT INSPECTION ROBOT IN CABLE TUNNEL
}

\author{
Shuangde Huang ${ }^{1}$, Zigeng Zhou' ${ }^{2}$, Baoyu $\mathrm{Xu}^{3}$, Shengwei Wang ${ }^{4}$, Tao Wang ${ }^{5}$ \\ 1-5 Kunming Power Supply Bureau of Yunnan Power Grid Co., Ltd., \\ Kunming, Yunnan 650011, China \\ shuangde h@outlook.com,zigzzzg@163.com, xbhbff36@163.com, \\ tswhs607@163.com, tzt8ck@sina.com
}

\begin{abstract}
Intelligent inspection robot is efficient for cable tunnel inspection. In this study, the path planning and positioning technology of inspection robot was studied. A new path planning technology based on Floyd algorithm and simulated annealing was proposed. The local shortest path was searched using Floyd algorithm, and then global shortest path was searched using simulated annealing algorithm. Radio frequency identification devices (RFID) combined with magnetic track guidance was used as positioning technology. Test of the positioning, straight driving and curve driving of intelligent inspection robot showed that the average error of the positioning technology, straight driving and curve driving was $7.1 \mathrm{~cm}, 4.2 \mathrm{~cm}$ and $5.6 \mathrm{~cm}$ respectively. It is concluded that the path planning and positioning technology proposed in this study has smaller errors, and will not affect the completion of inspection tasks. It provides a theoretical support for the application of intelligent inspection robot in cable tunnel.
\end{abstract}

Keywords: Cable Tunnel, Robot, Electric Inspection, Path Planning, Positioning and Navigation.

\section{Introduction}

With the development of economy and the acceleration of urbanization, underground cable tunnel as a new way of urban power grid construction has been more and more widely used. Underground cable tunnels can not only make full use of the limited land resources in cities, but also beautify the urban environment and reduce the impact of high-voltage transmission lines on the appearance of cities. The long-term operation of cable tunnel can not be separated from regular patrol inspection. At present, manual inspection is commonly used [1]. However, toxic and harmful gases in cable tunnels will pose a serious threat to the life safety of inspectors [2].

With the development of technology, intelligent inspection robot as a safe and efficient way of inspection has been more and more widely used in cable tunnels.

Path planning and automatic positioning are the key technologies of robots $[3,4]$, which have been deeply studied. Accurate and reliable positioning is a prerequisite for robots to accomplish tasks [5]. The positioning methods based on vision [6] and sensor [7] have been proved to be effective. In the aspect of path planning, Wei et al. [8] designed a path planning technology which combines the Internet of Things and fuzzy multi-objective decision-making.
It integrated the information of environmental constraints and path length into the fitness function, and the validity of the method was proved through simulation experiments. Mo et al. [9] proposed a path planning method combining biogeographic optimization (BBO), particle swarm optimization (PSO) and approximate Voronoi boundary network (AVBN). They used the position updating strategy of PSO to increase the diversity of population in BBO, and then the paths obtained by AVBN modeling were optimized. The simulation results showed that the method had high feasibility in robot path planning. Chaari et al. [10] proposed a tabu search planning algorithm for robot path planning.

The simulation experiments on small, medium and large grids showed that this method could find the optimal solution in small-scale environment and provide approximate optimal solution for large-scale maps with short execution time, suggesting a good performance in path planning. In this study, Floyd algorithm was combined with simulated annealing algorithm to realize the path planning of inspection robot, and then radio frequency identification devices (RFID) was combined with magnetic track guidance to realize robot positioning.

The effectiveness of this method was proved by experiments. This work provides some theoretical supports for the application of these technologies in the inspection of intelligent inspection robot in cable tunnel. 


\section{Intelligent Inspection Robot and its Navigation System}

Underground cable tunnel has a complex environment. Generally speaking, cable tunnels are ten to dozens of meters under the ground, with narrow space, long length, humid air and high temperature, and interference from small animals also exists. Compared with overhead cables, cable tunnels are more prone to aging, corrosion and damage. The corrosion and destruction of cables is easy to cause fire because of electricity mixing. The fire will spread quickly and the rescue will be difficult, which will cause large-scale power outage and cause huge economic losses. Hazardous substances after combustion will further corrode cables.

In order to ensure stable power transmission, it is necessary to maintain and inspect cable tunnel. At present, the inspection of cable tunnel is mostly carried out manually. However, due to the particularity of cable tunnel environment, manual inspection is difficult to detect fire hazards early and the efficiency is low. Moreover the safety of manual inspection is very low. Heat generated by cable working, harmful gases produced by smoldering, low oxygen content of underground tunnel and some seepage area may endanger the health and safety of inspectors, and the communication condition in tunnels is poor. Once an accident occurs, it will pose a great threat to the personal safety of inspectors.

With the rapid development of underground cable tunnels, it is more and more urgent to find a new efficient inspection method to replace manual inspection. The emergence of intelligent inspection robot effectively solves various disadvantages of manual inspection. Intelligent inspection robot can work all day and adapt to complex environment in tunnels and has high safety.

The intelligent inspection robot system can be divided into two parts: navigation and detection. An excellent navigation system can realize the function of path planning and autonomous positioning and make reasonable planning of its inspection route. When multiple robots work together, different working sections are assigned to the robot through positioning and route planning, so as to improve the inspection efficiency. When the robot detects any abnormal situation in cable tunnels, maintenance personnel will be arranged according to the positioning.

In this study, the inspection robot designed by Zhe Jiang Dali Technology Co., Ltd., China, (Figure 1) was taken as the research subject, and the path planning and positioning technology in its navigation system were designed. The robot was equipped with LMS511 laser scanner, which can build a global map of cable tunnel through laser scanning, serving for path planning and positioning.

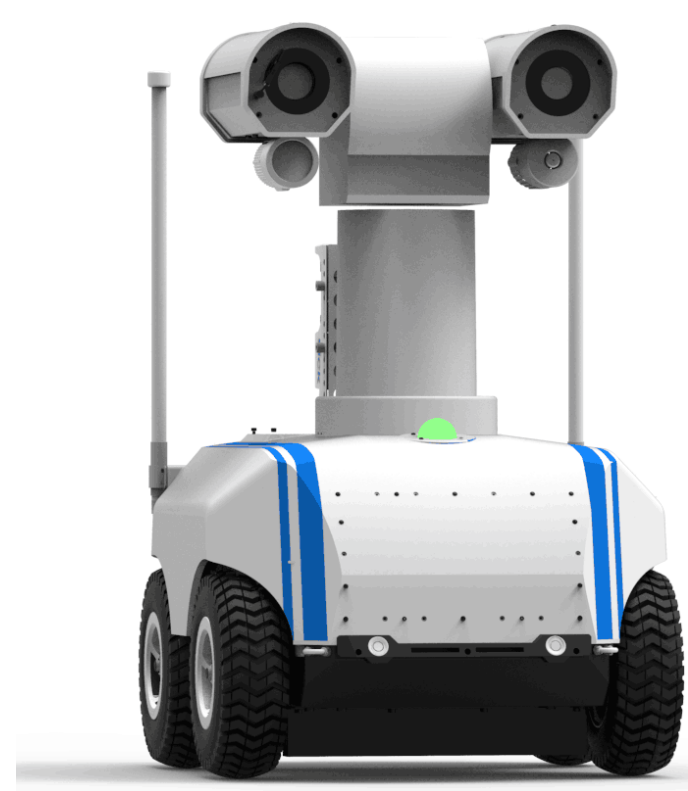

Figure 1: The inspection robot

\section{Path Planning and Positioning Technology of the Inspection Robot}

\section{- Path planning technology based on Floyd algorithm and simulated annealing}

Path planning technology of inspection robot is to find a passable and shortest path between the current position, the position that must be passed and the target position of the robot, so that the robot can complete as many inspection tasks as possible in the shortest time. According to the above requirements, the path planning technology not only needs to find the shortest path between two locations, that is, the local shortest path, but also needs to find the best combination between the locations to ensure the overall shortest path, that is, the global shortest path. Floyd algorithm is a classical path search method, which can find the shortest path between two points in a directed graph [11]. Simulated annealing algorithm is an intelligent search method, which can solve the optimization combination problem [12]. Therefore, Floyd algorithm was used for searching the local shortest path, and then simulated annealing algorithm was used for searching the global shortest path. The global optimal path planning was realized by the two algorithms.

\section{- Local shortest path searching based on Floyd algorithm}

The idea of Floyd is as follows. For two positions $V_{i}$ and $V_{j}$, the shortest distance $d_{i j}$ between the two positions is calculated. $d_{i j}$ is initialized. 
If the two positions are the same, then $d_{i j}=0$. If the two positions are adjacent, then $d_{i j}$ is the weight of edge between the two positions. If the two positions are not adjacent, $d_{i j}$ can be expressed as $\infty$. Adjacent matrix is established through $d_{i j}$. A new vertex is added to the original route between the two positions as the intermediate vertex. If the length of the path combination is smaller than the original path after the addition of the intermediate vertex, then the original path was replaced by the new path, and the matrix element is revised.

In the local shortest path searching of robot, suppose that map $M$ has $N$ points. $d_{i j}$ was initialized. Weighted adjacency matrix $A$ of $M$ is established, $A^{0}=\left(d_{i j}^{0}\right)_{N \times N}$. Firstly, the distance between $V_{i}$ and $V_{j}, d_{i 1}^{0}+d_{1 j}^{0}$, was calculated taking $V_{1}$ as the intermediate point. Suppose $d_{i j}^{1}=\min \left\{d_{i j}^{0},\left(d_{i 1}^{0}+d_{1 j}^{0}\right)\right\} . A^{1}=\left(d_{i j}^{1}\right)_{N \times N}$ was established. Then the distance between $V_{i}$ and $V_{j}, d_{i 2}^{1}+d_{2 j}^{1}$, was calculated taking $V_{1}$ and $V_{2}$ as the intermediate points. Suppose $d_{i j}^{2}=\min \left\{d_{i j}^{1}\left(d_{i 2}^{1}, d_{2 j}^{1}\right)\right\} . \quad A^{2}=\left(d_{i j}^{2}\right)_{N \times N}$ was established. The rest could be done in the same manner. The distance between $V_{i}$ and $V_{j}$, $d_{i n}^{n-1}+d_{n j}^{n-1}$, was calculated taking $V_{1}, V_{2}, V_{3} \ldots V_{n}$ as the intermediate points. Suppose $d_{i j}^{n}=\min \left\{d_{i j}^{n-1},\left(d_{i n}^{n-1}, d_{n j}^{n-1}\right)\right\} . \quad A^{n}=\left(d_{i j}^{n}\right)_{N \times N} \quad$ was established. $A^{n}$ was the shortest path matrix.

- Global shortest path searching based on simulated annealing algorithm

Simulated annealing algorithm is an optimization combination random search method simulating solid physical annealing process. It can avoid local optimal. It is a simple and flexible algorithm.

In the global shortest path searching of robot, suppose temperature as $T$, Markov chain length as $L$ and initial iteration times $\mathrm{K}$ as 0 . The specific steps of annealing algorithm are as follows.

$T, L$ and $K$ were initialized. A combination sequence of the initial position, target position and all positions which needed to be passed was randomly selected to express initial solution $X$, and energy function $E(X)$ was calculated.

Two positions which must be passed were exchanged, and then a new solution $X^{\prime}$ was obtained. Energy function $E\left(X^{\prime}\right)$ was calculated.

Difference value $\Delta E=E\left(X^{\prime}\right)-E(X)$ was calculated. If $\Delta E<0$, the new solution $X^{\prime}$ was accepted; otherwise the probability of acceptance was calculated using the formula: $P=\left\{\begin{array}{c}1, E\left(X^{\prime}\right) \leq E(X) \\ e^{-\frac{\Delta E}{T}}, E\left(X^{\prime}\right)>E(X)\end{array}\right.$. If random probability $P_{t}<\exp \left(-\frac{\Delta E}{t}\right)$, then $X^{\prime}$ was regarded as the new solution; otherwise $X$ was retained.

(4)When the termination condition was satisfied, the optimal path was output, and the algorithm ended.

The flow of robot path planning based on Floyd algorithm and simulated annealing algorithm is shown in Figure 2.

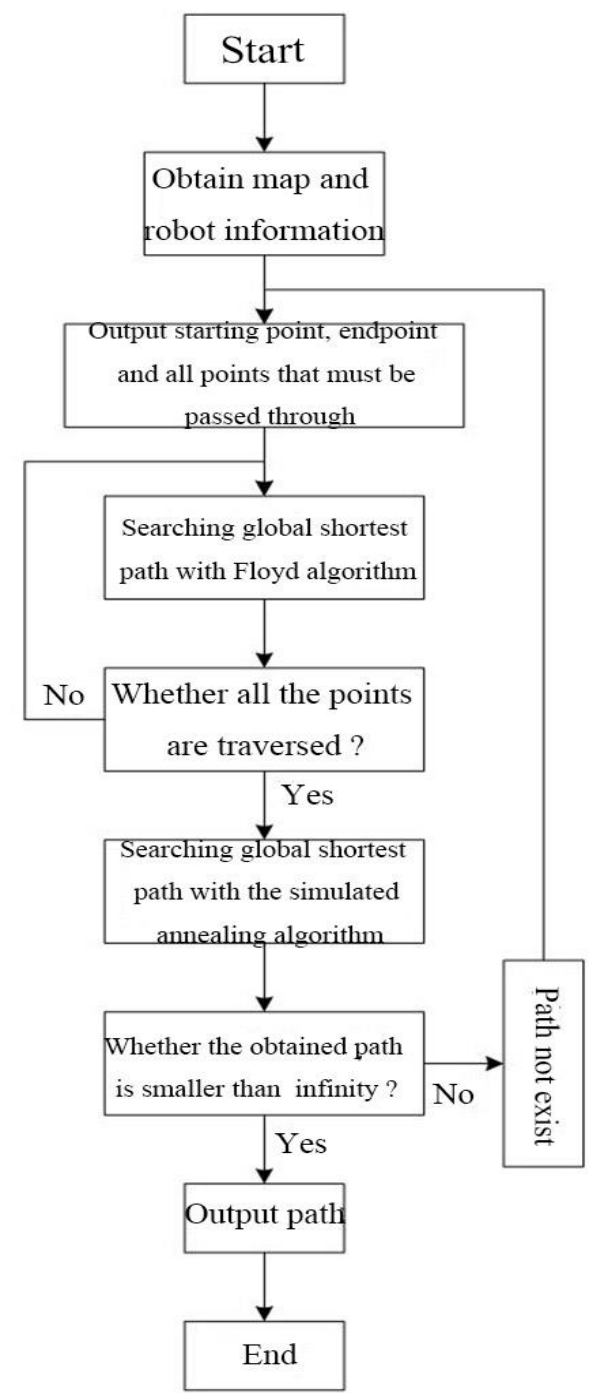

Figure 2: The flow of robot path planning

- RFID and magnetic track guidance based positioning technology

Robot positioning technology adopted RFID in combination with magnetic track guidance. Magnetic stripes were paved on the road that the robot passed by. The robot regarded magnetic stripes as the signal source. The magnetic field information was obtained by internal magnetic 
sensors to ensure that the trajectory of the robot was consistent with the magnetic trajectory. Magnetic navigation is an excellent navigation way, and it will not be disturbed in the harsh environment of cable tunnel. RFID technology was used in the identification of positions.

When the robot reached the position of the RFID tag, the information was collected and transmitted to the robot system to realize the positioning of the robot.

\section{Robot Path Planning and Positioning Test}

The flow of the intelligent robot navigation is obtained based on the shortest path planning and positioning, as shown in Figure 3.

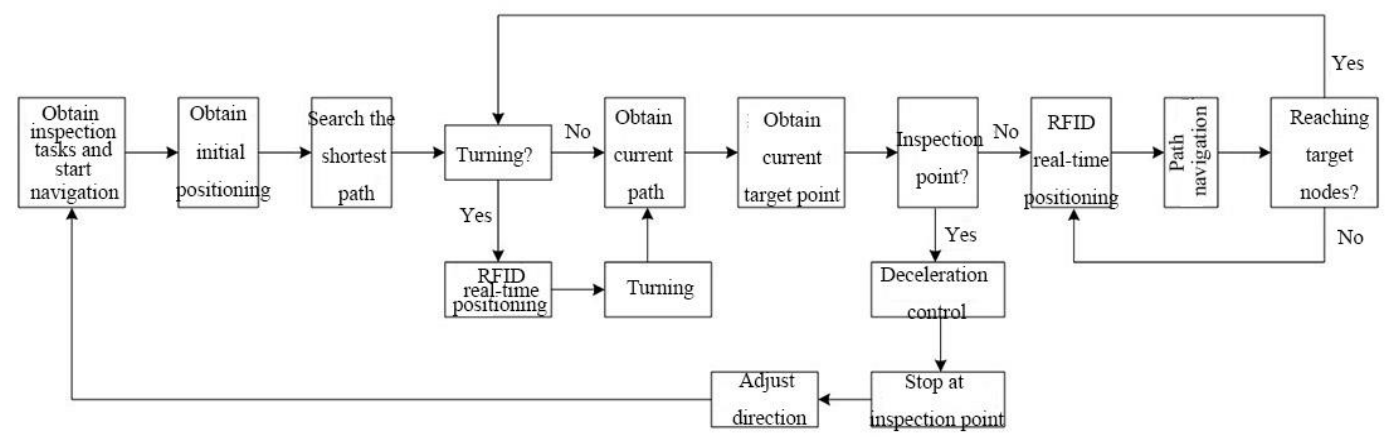

Figure 3: The flow of the robot navigation

After the robot received the inspection task, positioning initialization started, and the current location information was obtained using the positioning technology which combined RFID with magnetic trajectory guidance. Then the shortest path was calculated according to the target position. Firstly, the shortest path was searched using Floyd algorithm. Then simulated annealing algorithm was used to obtain the global shortest path. Whether the robot needed to turn was determined. After turning, the current path and position were obtained again. After reaching the inspection point, the robot was controlled to decelerate, stop at the inspection point, adjust the direction again after completing the inspection task, and resume the inspection.

\section{- Robot positioning test}

Firstly, the positioning test was carried out. Four target points were marked on the map of cable tunnel. The robot moved to the target point from the starting point repeatedly. The actual and target positioning of the robot were compared through the results of RFID positioning, and the positioning error was calculated. The results of 20 times of positioning are shown in Figure 4.

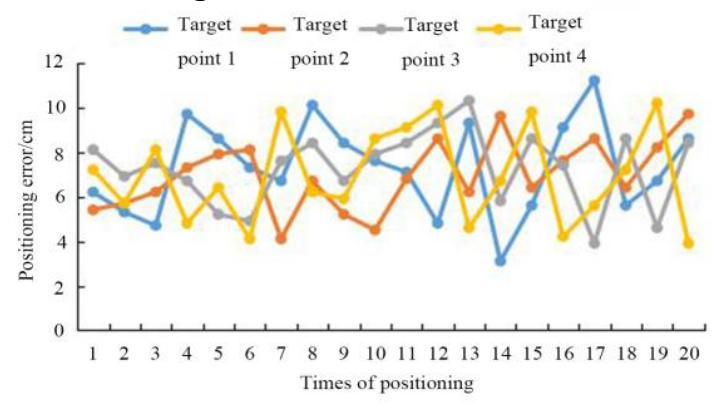

Figure 4: The error of the inspection robot positioning
It was found from Figure 4 that the positioning error of the robot was within $10 \mathrm{~cm}$, the maximum error was $11.2 \mathrm{~cm}$, the minimum error was $3.1 \mathrm{~cm}$, and the average error was $7.1 \mathrm{~cm}$. It showed that the intelligent inspection robot based on RFID positioning technology could realize accurate positioning in the process of cable tunnel inspection, with small positioning error and high positioning accuracy.

\section{- Robot straight path planning test}

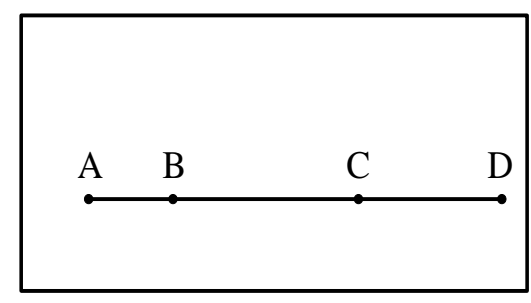

Figure 5: Robot straight driving path

The robot completed the inspection path as shown in Figure 5. A and B represent the path nodes and $\mathrm{C}$ and $\mathrm{D}$ represent the inspection points. Point $\mathrm{A}$ is the starting point, point $\mathrm{D}$ is the target position, and the speed of the robot was $200 \mathrm{~mm} / \mathrm{s}$. The straight driving condition of the robot was tracked.

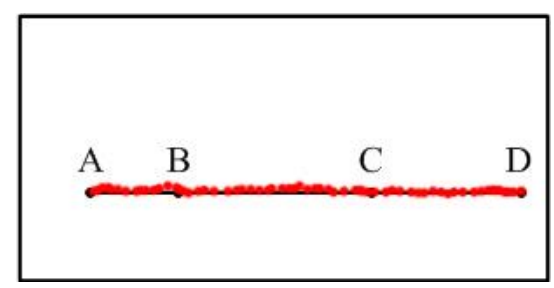

Figure 6: The track of the robot in the straight driving 
Figure 6 shows the tracking result of the intelligent inspection robot in straight driving. Straight line represents the established path, and red represents the position information of the robot in the actual driving process according to the path planned based on Floyd algorithm and simulated annealing algorithm. It was found that the actual driving path of the robot basically coincided with the established path, indicating that the path planning technology had a good performance in the straight driving process of the robot.

Table 1. Error analysis of the straight driving

\begin{tabular}{|l|l|l|l|}
\hline & $\begin{array}{l}\text { Maximum } \\
\text { error }\end{array}$ & $\begin{array}{l}\text { Minimum } \\
\text { error }\end{array}$ & $\begin{array}{l}\text { Average } \\
\text { error }\end{array}$ \\
\hline Error $(\mathrm{cm})$ & 7.1 & 0 & 4.2 \\
\hline
\end{tabular}

Table 1 shows the error of the robot in the straight line. The maximum error between the actual path and the fixed path was $7.1 \mathrm{~cm}$, the minimum error was $0 \mathrm{~cm}$, and the average error was $4.2 \mathrm{~cm}$. It was found that the robot had very small error in the straight driving, which showed that the path planning played a very good role.

\section{- Robot curve path planning test}

The curve driving of the robot was tested. The robot carried out inspection along the path as shown in Figure 7. The track of the robot in the curve driving is shown in Figure 8.

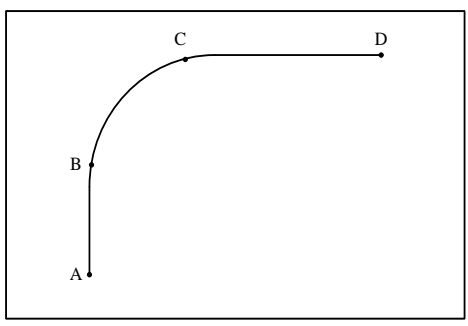

Figure 7: The curve driving path of the robot

Red in Figure 8 represents the position information of the robot in actual driving under the guidance of path planning. It was found that the position of the robot basically coincided with the established path in the actual driving process, it could accurately stop at the inspection point, and the difference between the actual path and the established path was relatively large in the process of turning.

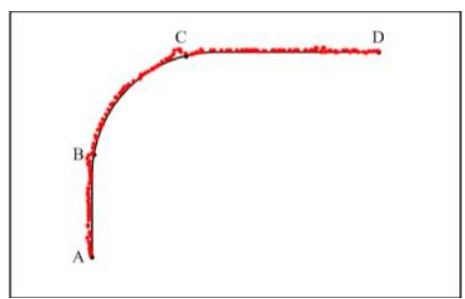

Figure 8: The track of the robot in the curve driving
Table 2. Error analysis of the curve driving

\begin{tabular}{|l|l|l|l|}
\hline & $\begin{array}{l}\text { Maximum } \\
\text { error }\end{array}$ & $\begin{array}{l}\text { Minimum } \\
\text { error }\end{array}$ & $\begin{array}{l}\text { Average } \\
\text { error }\end{array}$ \\
\hline Error $(\mathrm{cm})$ & 10.3 & 0 & 5.6 \\
\hline
\end{tabular}

The error analysis in Table 2 shows that the position error of the robot was larger in the curve driving than in the straight driving. The maximum error was $10.3 \mathrm{~cm}$ and the average error was $5.6 \mathrm{~cm}$, but the overall error was small, which had no influence on the completion of inspection tasks.

The analysis of the straight and curve driving of the robot suggested that the path planning technology could plan the driving path of the robot very well, with a small error, and could help the robot to complete inspection tasks quickly and efficiently.

\section{Discussion}

The rapid development of economy has put forward new requirements for power system [13]. More and more transmission lines are laid by cable tunnel. Cable tunnel has become the main power supply mode in more and more large cities. In order to ensure the normal operation of cable tunnels, regular inspection of cable tunnels is needed. At present, the commonly used manual inspection method is inefficient and dangerous [14]. With the development of technology, intelligent patrol robot has been well applied in cable tunnel. It can replace manual inspection and conduct more efficient and safe inspection. It is the development mode of cable tunnel inspection in the future. Path planning is the most important part of robot autonomous navigation [15]. This study mainly explored the path planning technology and positioning technology in navigation system.

Floyd algorithm is a good algorithm for searching the shortest distance between two locations. In this study, it was applied in the searching of the local shortest path of the robot.

Then in order to obtain the global shortest path, simulated annealing algorithm was selected to obtain the shortest inspection path, thereby improving the inspection efficiency. As to positioning technology, RFID combined with magnetic track guidance was used to achieve rapid and accurate positioning of the robot, so as to obtain cable fault information and timely remove faults.

In order to verify the validity of the method, the positioning, straight driving and curve driving of the robot were tested respectively. The analysis of the positioning error in Figure 4 indicated that the positioning error fluctuation of the robot was small, the error value was small, basically within $10 \mathrm{~cm}$, the maximum error was $11.2 \mathrm{~cm}$, the minimum error was $3.1 \mathrm{~cm}$, and the average error was $7.1 \mathrm{~cm}$. 
It showed that the positioning technology had small positioning error and could accurately locate the robot, i.e., the method was effective. Figure 6 and 8 showed that the actual track of the robot coincided with the established path basically in the straight and curve driving, and the overall error was small. It showed that the robot could inspect along the established path with the help of path planning and positioning technology and stop at the inspection point to complete the inspection task. The average error of straight driving was $4.2 \mathrm{~cm}$, and that of curve driving was $5.6 \mathrm{~cm}$. Figure 8 shows that the error of robot was relatively large at the path junction. Curve path planning of robots is very difficult, and it is always an important and difficult problem in the field of robot navigation.

The path planning technology proposed in this paper can control the errors of straight and curve driving in a small range, but further research is needed to improve the navigation accuracy of curve driving.

\section{Conclusions}

In this study, the path planning and positioning technology of intelligent inspection robot was studied. Floyd algorithm and simulated annealing algorithm were used for path planning, and RFID technology and magnetic track guidance were used for high-precision positioning. The validity of the method was proved by test, providing some theoretical supports for the better application of these technologies in the inspection of cable tunnels. This work is helpful to promote the further development of intelligent inspection robot.

\section{Acknowledgements}

The study was supported by "Science and Technology Project of China Railway Corporation, China (Grant No. 1341324011)".

\section{References}

[1] Montero R, Victores J G, Martínez S, Jardón A, Jardón C. "Past, Present and Future of Robotic Tunnel Inspection. Automation in Construction," 2015, 59:S0926580515000229.

[2] Yang Z, Zou P, Wu G, Gao J, Song W, Peng S, Zhou Q. "Fabrication and Characterization of Highly Sensitive Methane Sensor Based on Titanium Dioxide," Journal of Nanoelectronics \& Optoelectronics, 2017, 12(8):815-819.
[3] Sobreira H, Pinto M, Moreira A P, Costa P G, Lima J. "Robust Robot Localization Based on the Perfect Match Algorithm," Lecture Notes in Electrical Engineering, 2015, 321:607-616.

[4] Hossain M A, Ferdous I. "Autonomous Robot Path Planning in Dynamic Environment Using a New Optimization Technique Inspired by Bacterial Foraging Technique," Robotics and Autonomous Systems, 2015, 64:137-141.

[5] Hornung A, Oßwald S, Maier D, Bennewitz M. "Monte Carlo Localization for Humanoid Robot Navigation in Complex Indoor Environments," International Journal of Humanoid Robotics, 2014, 11(02):1441002.

[6] Ran L, Zhang Y, Zhang Q, Yang T. “Convolutional Neural Network-Based Robot Navigation Using Uncalibrated Spherical Images," Sensors, 2017, 17(6):1341.

[7] Wu D, Chatzigeorgiou D, Youcef-Toumi K, BenMansour R. "Node Localization in Robotic Sensor Networks for Pipeline Inspection," IEEE Transactions on Industrial Informatics, 2016, 12(2):809-819.

[8] Wei L S, Guo Y, Dai X F. "Path Planning Based on Warehousing Intelligent Inspection Robot in Internet of Things," Advanced Materials Research, 2011, 267:318-321.

[9] Mo H, Xu L. "Research of biogeography particle swarm optimization for robot path planning," Neurocomputing, 2015, 148:91-99.

[10] Chaari I, Koubaa A, Bennaceur H, Ammar A, Trigui S, Tounsi M, Shakshuki E, Youssef H. "On the Adequacy of Tabu Search for Global Robot Path Planning Problem in Grid Environments," Procedia Computer Science, 2014, 32:604-613.

[11] Lin Z, Jianli Z. "Frequent Item Sets and Association Rules Mining Algorithm Based on Floyd Algorithm," Journal of Computational and Theoretical Nanoscience, 2015, 12(9):25742578.

[12] He X S, Ding W J, Yang X S. "Bat Algorithm Based on Simulated Annealing and Gaussian Perturbations," Neural Computing and Applications, 2014, 25(2):459-468.

[13] Qin X, Wu G, Lei J, Fan F, Ye X, Mei Q. "A Novel Method of Autonomous Inspection for Transmission Line based on Cable Inspection Robot LiDAR Data," Sensors, 2018, 18(2):596.

[14] Attard L, Debono C J, Valentino G, Di Castro M. "Vision-based Change Detection for Inspection of Tunnel Liners," Automation in Construction, 2018, 91:142-154.

[15] Thoa Mac T, Copot C, Tran D T, De Keyser R. "Heuristic Approaches in Robot Path Planning: A Survey," Robotics and Autonomous Systems, 2016:S0921889015300671. 